\title{
Integrating a Clinical Decision Support Reminder to Improve Blood Pressure Reassessment for Patients with Uncontrolled Hypertension
}

\author{
K. Souffront ${ }^{1 *}$, L. Rivera², G. Loo ${ }^{3}$, N. Genes ${ }^{4}$, P. L. Richardson ${ }^{5}$ Levy $^{6}$ \\ 1.2.3.4.5 Ichan School of Medicine at Mount Sinai/Mount Sinai Hospital, Department of Emergency Medicine, New York, NY \\ ${ }^{6}$ Wayne State University, Department of Emergency Medicine, Detroit, MI
}

*Corresponding Author : K. Souffront, Ichan School of Medicine at Mount Sinai/Mount Sinai Hospital, Department of Emergency Medicine, New York, NY.

Received date: February 01, 2019;Accepted date : February 26, 2019; Published date: March 19, 2019.

Citation : K. Souffront, L. Rivera, N. Genes, P. L. Richardson, Levy, Integrating a clinical decision support reminder to improve blood pressure reassessment for patients with uncontrolled hypertension, J clinical Cardiology and Cardiovascular Interventions.

Doi: 10.31579/2641-0419/013

Copyright : @ 2019 K. Souffront. This is an open-access article distributed under the terms of The Creative Commons Attribution License, which permits unrestricted use, distribution, and reproduction in any medium, provided the original author and source are credited.

\begin{abstract}
Background: Emergency department clinicians often overlook asymptomatic hypertension (HTN). Clinical decision support can help improve adherence to the emergency nursing and emergency medicine clinical policy for asymptomatic HTN. While the policy indicates referral for all adults with two or more elevated blood pressures, less than $10 \%$ of patients are referred. We sought to determine the efficacy of an electronic health record clinical reminder on nursing (RN) reassessment of blood pressure (BP) for hypertensive patients.
\end{abstract}

Methods: We conducted a 2-arm, pilot RCT, at an academic medical center in New York City. 107 RNs were randomized to the control group or to the intervention of a 'Best Practice Alert' (BPA) reminding him/her to recheck the BP in adult patients with an initial BP reading $\geq 140 / 90 \mathrm{mmHg}$. Descriptive statistics that included univariate and bivariate analyses were used to obtain adjusted measures of association between the intervention and control group.

Results: RNs were more likely to repeat BP after receiving a BPA alert (56\%) compared to RNs who did not receive an alert (44\%) (OR=2.3, $\mathrm{Cl} 2.1-2.5 ; \mathrm{p}<.001)$. Patients who received $\mathrm{BP}$ reassessment were more likely to be triaged category $4(\mathrm{OR} 2.88, \mathrm{Cl} 1.81-4.59, \mathrm{p}=.0001) ;$ age $>75$ years (OR 1.47; Cl 1.07-2.03; $p=.02$ ); had Stage II HTN (OR 3.48; Cl 2.63-4.59, $p=.0001)$ and an ED length of stay of 3-4 hours (OR 5.85; $\mathrm{Cl}$ 4.43-7.73; $\mathrm{p}=.0001)$.

Conclusion: The BPA alert was effective in increasing BP reassessment by ED nurses. The findings of this study will help us translate this evidence ED practices.

\section{Introduction}

Hypertension is the most common cardiovascular disease (CVD) in the United States (U.S.), and as such, a frequently encountered condition among patients who visit the emergency department (ED). ${ }^{1}$ Professional emergency nursing (ENA) and emergency medicine (ACEP) organizations have endorsed efforts to better screen for HTN and help link those with uncontrolled BP to primary care. ${ }^{1}$ They recommend referral for all adults in which their BP is persistently elevated (two or more BP readings $\geq 140 / 90 \mathrm{~mm} \mathrm{Hg}$ ). Unfortunately, despite clear recommendations from their professional societies, less than $10 \%$ of patients are referred. ${ }^{1}$ The Centers for Medicaid and Medicare Services (CMS) recently adopted a measure (\#317), which imposes a financial penalty for not referring patients with elevated BP to primary care, underscoring the importance of this issue. ${ }^{2}$ Improved vigilance identifying high-risk patients with HTN may be achieved by implementing novel, multi-disciplinary, streamlined interventions.

Operationally, improving BP management in the ED requires three steps: 1) nursing BP assessment and reassessment, 2) provider referral for patients who have persistent HTN, and 3) successful linking of patients to outpatient management. Appropriate follow-up after BP measurement is a pivotal component in preventing the progression of HTN and the development of heart disease. A recent retrospective analysis conducted by the first and last author demonstrated our BP reassessment rate after an initial abnormal reading was significantly low (49\%). 2 We sought to improve nurse BP reassessment, using a clinical decision support (CDS) alert integrated into an existing electronic health record (EHR) platform (EPIC), serving as the first and vital step for improving BP management in the ED.
Real-time CDS algorithms may help improve adherence to the ENAendorsed and ACEP Policy. CDS interventions have been shown to be effective in a variety of settings, including the ED.1 However, no CDS tool to improve adherence to the ACEP Policy for asymptomatic HTN has been studied.

After a series of discussions with informatics experts and theoretical discussions about the barriers/facilitators to implementation with ED nurses and meaningful use, we pilot tested a randomized controlled trial (RCT) with ED nurses to improve BP reassessment. The purpose of our study was to determine the efficacy of an EHR clinical reminder on nursing BP reassessment for patients who were found to be hypertensive on initial ED triage vital signs.

\section{Methods}

Study Design: This was a 2-arm (intervention/control); randomized controlled pilot trial to determine the acceptability and efficacy of a CDS tool in the form of a clinical reminder to nurses, on BP reassessment.

Study Setting: Our study took place in the ED of a 1,174-bed tertiary care academic referral center located in the upper east side of New York City, which sees over 100,000 visits per year and serves a diverse population that is $35 \%$ Latino, 29\% Black, $19 \%$ White, $17 \%$ Asian \& other. The ED uses a single electronic health record (EPIC).

Study Sample: We included all RNs $(n=107)$ (Full-Time, Part-Time, Per-diem). We included patient's $\geq 18$ year old who had an initial systolic $\mathrm{BP} \geq 140 \mathrm{mmHg}$ or an initial diastolic $\mathrm{BP} \geq 90 \mathrm{mmHg}$. The initial systolic/diastolic BP mean and standard deviation for the study sample was 
$162( \pm 22.1) / 84( \pm 13.4)$. 
Study Protocol: RNs were randomized to the intervention $(n=54)$ or control group $(n=53)$. Random assignment was performed by a computer generated random number list using Excel 2007 (Microsoft, Redmond, WA, USA), where nurses were allocated to either intervention or control groups. Nurses remained in their assigned group for the entire study period. Patients with initial BP $\geq 140 / 90 \mathrm{~mm} \mathrm{Hg}$ triggered the CDS electronic reminder to repeat BP. This CDS tool integrated into the EHR was in the form of a "Best Practice Alert" (BPA) that displayed a message that 1) reminded the $\mathrm{RN}$ of the ACEP/ENA-endorsed clinical policy, and 2) prompted the RN to document BP reassessment; a link directly into the vital signs flow sheet where the BP is entered was provided. This alert was shown each time the Registered Nurse went into the chart until a second BP was documented. At 12 weeks, we performed our analysis to evaluateour main outcome, BP reassessment.

Data collection: We electronically extracted data for BP reassessment, which was defined as any repeat BP (yes/no). In 2010, there were approximately 21,000 discharges from our ED, of patients with a $\mathrm{BP}$ reading $\geq 140 / 90 \mathrm{mmHg}$, translating to $\sim 5200$ patient encounters over the three-month data collection period. Using a basal rate of $\mathrm{BP}$ reassessment $=50 \%$ would yield sufficient power to conduct our study with rigor across a range of projected effect sizes.

Data Analysis: First, we described independent variables (provider and patient) in percentages (\%) or means (M) and standard deviations (SD) and compare the percentage of patients with BP reassessment between the intervention group and control group. Next, bivariate analyses were conducted using chi-square tests to test for significant relationships between provider and patient demographics and BP reassessment. Next, a multiple logistic regression analysis was performed. The best-fit model predictive of BP reassessment was produced and presented in.

\begin{tabular}{|l|l|l|}
\hline Demographic Variable & Overall & Statistics (p-value) \\
\hline CDS Alert & & 0.0001 \\
\hline o Yes & $3,611(22.05 \%)$ & \\
\hline o No & $12,767(77.95 \%)$ & \\
\hline Gender, male & $9681(59.1 \%)$ & 0.0001 \\
\hline Age, n (\%) & & 0.0001 \\
\hline o 18-24 & $2477(15.2 \%)$ & \\
\hline o 25-44 & $5869(35.8 \%)$ & \\
\hline o 45-64 & $5171(31.6 \%)$ & \\
\hline o 65-74 & $1421(8.7 \%)$ & \\
\hline o 75+ & $1440(8.8 \%)$ & \\
\hline Race & & 0.0001 \\
\hline o Black & $6105(37.28)$ & \\
\hline o White & $2398(14.64)$ & \\
\hline o Other & $6768(41.32)$ & \\
\hline Ethnicity, Hispanic & $9021(59.17)$ & 0.0001 \\
\hline HTN History & $5094(31.1 \%)$ & 0.0001 \\
\hline Repeated BP & $6563(40.1 \%)$ & 0.0001 \\
\hline ESI, n (\%) & & 0.0001 \\
\hline o 5 Non Urgent & $576(3.54)$ & \\
\hline o 4 Less Urgent & $4479(27.49)$ & \\
\hline o 3 Urgent & $8813(54.09)$ & \\
\hline o 2 Emergent & $2392(14.68)$ & \\
\hline o 1 Immediate & $32(0.2)$ & \\
\hline & & \\
\hline fle 1. Den $)$ & \\
\hline
\end{tabular}

Table 1. Demographic description of all unique patient encounters over the 12-week study period.

\begin{tabular}{|l|l|l|l|l|}
\hline Results. & B & OR & 95\% CI & P-value \\
\hline Covariate & \multicolumn{5}{|l|}{} \\
\hline BPA Alert & 1.057 & 2.88 & $1.81,4.59$ & 0.0001 \\
\hline ESI Urgent & \multicolumn{1}{l|}{} \\
\hline BP Category & REF & REF & REF & REF \\
\hline o Normal & 0.229 & 1.26 & $1.04,1.52$ & 0.02 \\
\hline o Borderlin & 0.565 & 1.76 & $1.36,2.27$ & 0.0001 \\
\hline o Stage I HTN & 1.245 & 3.48 & $2.63,4.59$ & 0.0001 \\
\hline o Stage II HTN & 0.0386 & 1.47 & $1.07,2.03$ & 0.02 \\
\hline Age >75 years & & 1.35 & $1.00,1.82$ & 0.05 \\
\hline ED LOS & 0.30 .1 & $1.74,3.10$ & 0.0001 \\
\hline o 1-2 hours & 0.844 & 2.37 & 1.74 \\
\hline o 2-3 hours & 5.85 & $4.43,7.73$ & 0.0001 \\
\hline o 3-4 hours & & 517 & \\
\hline AIC
\end{tabular}

\section{Table 2.}

As shown in Table 2 a greater proportion of patients were male (59.1\%), self-identified as Black (37.28\%), Hispanic (59.17\%), triaged as ESI category $3(54.09 \%)$.

\section{Discussion}

Implementation of our CDS alert was derived from the 2006 ACEP (and ENA-endorsed) clinical policy, which recommends BP reassessment for an initial abnormal BP reading $\geq 140 / 90 \mathrm{mmHg}$ and referral to PCP for persistent asymptomatic HTN. ${ }^{3}$ While ACEP policies have been shown to be safe and effective, increasing ED clinician adherence to this policy has been an ongoing challenge. ${ }^{45}$ We sought to improve nurse BP reassessment, using a CDS alert, as an important first step for improving adherence to this clinical policy. CDS has been integrated into EHRs to enhance nursing decision-making and evidence- based practice. ${ }^{6}$ In a 2012 AHRQ Evidence Report/Technology Assessment on CDS and knowledge management, Lobach et al. identified 15,176 citations,including 1,407 full text articles. ${ }^{7}$ The meta-analysis revealed strong evidence that CDS can improve process outcomes, including increased preventive services with an odds ratio of 1.42 (95\% CI $=1.27,1.58)$.

Results of our pilot RCT provide preliminary evidence that CDS, in the form of an electronic reminder, may improve nurse BP reassessment among patients who present to the ED with an initial abnormal BP reading. However, future modification of our EHR reminder is necessary to reinforce and sustain BP reassessment.

A systematic review of 70 studies documented features of CDS necessary for successful implementation. ${ }^{8}$ Results found four features of CDS to be independent predictors of improved clinical practice. These four features included provision of CDS (1) as part of clinician workflow, (2) with recommendations rather than assessments, (3) at the time and location of decision-making, and (4) that is computer-based. While the majority of these studies reviewed were physician-driven, we believe our nurse focused CDS tool adheres to these features.

In addition to key features, it will mostly likely take more trial and error in order to optimize penetration of our CDS into actual practice. For example, audit-feedback (AF) has been shown to improve adherence to clinical policies and thus, a mechanism to track BP reassessment after receiving an alert and provide individual feedback at the individual level may be considered as part of future interventions. ${ }^{9}$ A meta-analysis of $\mathrm{AF}$ and impact on effectiveness of care quality found that $\mathrm{AF}$ has a significant impact on quality outcomes $(\mathrm{d}=0.40,95 \% \mathrm{CI}=+/-0.20)$; providing specific suggestions for improvement, written, and more frequent feedback strengthened this effect. This was attenuated when $\mathrm{AF}$ was in the form of graphical or verbal display. ${ }^{9}$ While $\mathrm{AF}$ is one proven way to accelerate uptake of CDS, several authors have cited "alert fatigue", "integrated systems (being) too distractive", or the "systems being user hostile in some other way”, as reasons why CDS may have a negative impact. 
AHRQ describes "alert fatigue" occurs when clinicians become desensitized to alerts (because of the sheer volume), and as a result ignore or fail to respond to the alert/reminder.

Although we found that BP reassessment improved for our nurses who received the alert, we also found that ED LOS had a significant impact on $\mathrm{BP}$ reassessment in our final regression model, whether or not the ED nurse received a CDS alert. Clinical theory supports these findings, such that the longer a patient is in the ED the more likelythey are to have their BP reassessed at some time-point during the ED stay. ED mean LOS per patient measured from the patient's arrival to departure has been promoted as a surrogate indicator of crowding in the absence of a standard or universal definition. It is also frequently considered a key process indicator for performance improvement and clinical and operational efficiency.

\section{Conclusion}

In our 2-arm RCT, we found that a CDS, in the form of a clinical reminder, might improve BP reassessment among nurses. This study is the first of its kind and will move the field forward. This study serves as an important first step to promote adherence to clinical policy and patient care, especially, for a patient population that is disproportionately affected by uncontrolled HTN, ultimately helpingto close the gap in health disparities related to HTN.

\section{References}

1. Chobanian AV, Bakris GL, Black HR, et al. (2003) The Seventh Report of the Joint National Committee on Prevention, Detection, Evaluation, and Treatment of High Blood Pressure: the JNC 7 report. 289(19):2560-2572.

2. The Center for Medicare and Medicaid Physician Quality Reporting System. Accessed from June 8th 2016.
3. Decker WW, Godwin SA, Hess EP, Lenamond CC, Jagoda AS. Et al (2006) Clinical policy: critical issues in the evaluation and management of adult patients with asymptomatic hypertension in the emergency department. Annals of emergency medicine.47(3):237-249.

4. Souffront K, Chyun D, Kovner C. (2015) Barriers to referral for elevated blood pressure in the emergency department and differences between provider-type. Journal of clinical hypertension (Greenwich, Conn).17(3):207-214.

5. Souffront K, Gestal C, Melkus GD, Richardson L. (2016)Recognition of Asymptomatic Hypertension in an Urban Emergency Department: Where Are We Now? Advanced emergency nursing journal. 38(4):320326.

6. Whalen K, Bavuso K, Bouyer-Ferullo S, (2016) Analysis of Nursing Clinical Decision Support Requests and Strategic Plan in a Large Academic Health System. Applied clinical informatics. 7(2):227-237.

7. Lobach D, Sanders GD, Bright TJ, et al. Enabling health care decisionmaking through clinical decision support and knowledge management.Evidence report/technology assessment.2012(203):1-784

8. Kawamoto K, Houlihan CA, Balas EA, Lobach DF. Improving clinical practice using clinical decision support systems: a systematic review of trials to identify features critical to success. BMJ (Clinical research ed). 2005;330(7494):765.

9. Hysong SJ. (2009) Meta-analysis: audit and feedback features impact effectiveness on care quality. Medical care.47(3):356-363.

10. Wright JT, Jr., Williamson JD, Whelton PK, (2015) A Randomized Trial of Intensive versus Standard Blood-Pressure Control. The New England journal of medicine. 373(22):2103-2116.

11. Goldberg EM, Wilson T, Saucier C, et al. Achieving the BpTRUth: emergency department hypertension screening and the Centers for Medicare \& Medicaid Services quality measure. Journal of the American Society of Hypertension : JASH.2017;11(5):290-294. 